\title{
Efeito de lodo de esgoto na fertilidade de um Argissolo Vermelho-Amarelo cultivado com cana-de-açúcar(1)
}

\author{
Fábio Cesar da Silva( ${ }^{(2)}$, Antonio Enedi Boaretto(3), Ronaldo Severiano Berton ${ }^{(4)}$, \\ Helder Bazaglia Zotelli(5), Carlos Alberto Pexe ${ }^{(5)}$ e Elaíne Mendonça Bernardes ${ }^{(6)}$
}

\begin{abstract}
Resumo - O objetivo deste trabalho foi avaliar os efeitos da aplicação de 0, 20 e $40 \mathrm{Mg} \mathrm{ha}^{-1}$ de lodo de esgoto, na presença e ausência de fertilizante mineral (NPK) na fertilidade e nos teores de metais pesados de um Argissolo Vermelho-Amarelo distrófico, cultivado com cana-de-açúcar. Amostras de solo foram coletadas aos 146, 272 e 484 dias após o plantio da cultura de cana-de-açúcar e foram submetidas à análise de fertilidade pelo método do Instituto Agronômico de Campinas, incluindo a determinação do $\mathrm{S}, \mathrm{Cr}, \mathrm{Cd}, \mathrm{Cu}, \mathrm{Fe}, \mathrm{Mn}, \mathrm{Pb}$ e $\mathrm{Zn}$. O lodo de esgoto diminuiu a acidez do solo e forneceu $\mathrm{Ca}, \mathrm{P}, \mathrm{S}$ e Zn, principalmente. Seus efeitos foram de curta duração, restringindo-se a um ano agrícola Os teores dos metais pesados das amostras de solo tratado foram maiores que os da testemunha, mas menores que os valores considerados perigosos ao ambiente.
\end{abstract}

Termos para indexação: Saccharum officinarum, águas servidas, dejetos industriais, fertilidade do solo, metais pesados.

Effect of sewage sludge on the fertility of a Paleudult soil cultivated with sugarcane

\begin{abstract}
Effects of sewage sludge applied to sugarcane, alone $\left(0,20\right.$ and $\left.40 \mathrm{Mg} \mathrm{ha}^{-1}\right)$ or combined with mineral fertilizer (NPK), on a Paleudult soil fertility and on the soil heavy metal were evaluated. Soil samples were collected at 146, 272 and 484 days after the sugarcane was planted. Routine analysis for soil fertility evaluation using the resin extraction procedure was performed and $\mathrm{S}, \mathrm{Cr}, \mathrm{Cd}, \mathrm{Cu}, \mathrm{Fe}$, $\mathrm{Mn}, \mathrm{Pb}$ and $\mathrm{Zn}$ were also analyzed. Sewage sludge decreased soil acidity and was a source of $\mathrm{Ca}, \mathrm{P}, \mathrm{S}$ and $\mathrm{Zn}$, mainly, but its effects on soil properties lasted only one crop season. Sewage sludge application increased soil heavy metal contents but not to levels considered harmful to the environment
\end{abstract}

Index terms: Saccharum officinarum, wastewater, industrial wastes, soil fertility, heavy metals.

\section{Introdução}

As águas servidas, quando lançadas aos rios, constituem o principal agente de poluição em regiões com alta densidade demográfica e industrial. A solução

(1) Aceito para publicação em 2 de junho de 2000

(2) Embrapa-Centro Nacional de Pesquisa Tecnólogica em Informática para a Agricultura, Caixa Postal 6041, CEP 13083-970 Campinas, SP. Bolsista do CNPq. E-mail: fcesar@enptia.embrapa.br

(3)USP, Centro de Energia Nuclear na Agricultura, Caixa Postal 96, CEP 13400-970 Piracicaba, SP. Bolsista do CNPq.

(4) Instituto Agronômico, Caixa Postal 28, CEP 13001-970 Campinas, SP

(5)Usina Costa Pinto S/A, Caixa Postal 1205, CEP 13411-900 Piracicaba, SP.

${ }^{(6)}$ UNESP, Faculdade de Engenharia de Ilha Solteira, Dep. de Fitotecnia, Economia e Sociologia Rural, Rua Monção s/n, CEP 15385-000 Ilha Solteira, SP. que vem sendo adotada em diversos municípios é realizar o tratamento das águas servidas, de cujo processo se origina o lodo de esgoto (LE). Um destino adequado deve ser dado a esse resíduo e, para tal, algumas alternativas têm sido propostas. A disposição em aterros sanitários é uma opção aceitável tecnicamente, porém apresenta custo de manutenção muito elevado; o despejo nos oceanos, rios e lagos é prática proibida por lei nos Estados Unidos por causar sérios danos ao ambiente; a incineração é uma alternativa prática mas não econômica, pois gera gases poluentes para a atmosfera. $\mathrm{O}$ uso do LE na agricultura é a alternativa mais viável e interessante, pois o resíduo é fonte de matéria orgânica e de nutrientes para as plantas (Ros et al., 1991), e age como corretivo da acidez do solo (Berton et al., 1989; Dias, 1994). Assim, o uso de LE na adubação contribui para reduzir os gastos com fertilizantes, principalmente fosfatados e nitrogenados (Carvalho \& Barral, 
1981); além disso, com a aplicação do LE ao solo há retorno ao campo de parte dos nutrientes exportados às cidades na forma de produtos agrícolas. Todavia, o LE pode conter metais pesados, como $\mathrm{Cd}, \mathrm{Zn}, \mathrm{Mn}$, $\mathrm{Cu}, \mathrm{Cr}$, Ni e $\mathrm{Pb}$, que podem se acumular na cadeia trófica animal (Carvalho \& Barral, 1981; Bettiol et al., 1983). Entre esses metais, $\mathrm{Cd}, \mathrm{Cu}, \mathrm{Ni}$ e Zn são os que apresentam maior risco de contaminação ambiental (Logan \& Chaney, 1983; Valadares et al., 1983).

A exigência nutricional da cultura a ser instalada determina a quantidade de LE que deve ser usada, e os teores dos metais pesados presentes no LE limitam o número de aplicações do resíduo que o mesmo local pode receber (Mulchi et al., 1987).

Em países industrializados, o LE é usado como fertilizante alternativo há muito tempo, e por este motivo já foram realizadas muitas pesquisas sobre os efeitos, no solo, deste resíduo. Entretanto, os resultados, que geralmente foram obtidos em solos de clima temperado, dificilmente podem ser extrapolados para os solos ácidos de clima tropical.

Este trabalho teve como objetivo avaliar as alterações na fertilidade e no teor de alguns metais pesados de um solo Argissolo Vermelho-Amarelo distrófico, cultivado com cana-de-açúcar resultantes da aplicação de lodo de esgoto combinado ou não com fertilizantes NPK.

\section{Material e Métodos}

O experimento foi instalado em 5 de março de 1993 na Fazenda Santa Helena, pertencente à Usina Costa Pinto
S/A, situada no Município de Piracicaba, Estado de São Paulo, com clima característico do tipo Cwa (classificação de Köppen), num Argissolo Vermelho-Amarelo distrófico, abrupto a moderado, textura arenosa/média, denominado unidade Serrinha (Arenic Abruptic Paleudulf). As características químicas do solo antes da instalação do experimento são apresentadas nas Tabelas 1 e 2. Verificase que o solo tem acidez elevada e saturação por bases muito baixa; teores muito baixo de $\mathrm{P}$, baixo de $\mathrm{Mg}$ e médio de $\mathrm{Ca}$ e $\mathrm{K}$, conforme interpretação pelos critérios de Raij et al. (1996). Segundo os mesmos autores, os teores de $\mathrm{Mn}$ e Fe são altos, e os de $\mathrm{Cu}$ e Zn são médios. Pelos teores de metais pesados pode-se afirmar que se trata de solo sem contaminação, pois apresentavam-se em faixa comumente encontrada nos solos tropicais (Rovers et al., 1983; Mattiazzo-Prezotto, 1994)

Amostras do LE obtidas na Estação de Recuperação da Qualidade da Água (ERQ), de Barueri, SP, foram secadas a $65^{\circ} \mathrm{C}$, e obteve-se teor de água de $620 \mathrm{~g} \mathrm{~kg}^{-1}$. Incinerou-se o LE em mufla, para determinação do $\mathrm{C}$ total, e submeteu-se o LE a digestão sulfúrica, para determinar o $\mathrm{N}$ total (Sarruge \& Haag, 1974), obtendo-se 71 e $22 \mathrm{~g} \mathrm{~kg}^{-1}$ para $\mathrm{C}$ e N, respectivamente, o que dá uma relação $\mathrm{C} / \mathrm{N}$ igual a 3,2 . Os demais elementos contidos no resíduo secado a $65^{\circ} \mathrm{C}$ foram extraídos com água régia $\left(\mathrm{HCl}+\mathrm{HNO}_{3}: 1+3\right)$, em forno de microondas (Nieuwenhuize et al., 1991). A composição do LE, expressa em $\mathrm{g} \mathrm{kg}^{-1}$, foi a seguinte: $\mathrm{Al}=2,0 ; \mathrm{P}=10 ; \mathrm{K}=1,5$; $\mathrm{Ca}=11,5 ; \mathrm{Mg}=3,5 ; \mathrm{S}=10$ e $\mathrm{Fe}=50$. Os teores de outros elementos analisados, expressos em $\mathrm{mg} \mathrm{kg}^{-1}$, foram: $\mathrm{B}=8,5 ; \mathrm{Cu}=905 ; \mathrm{Zn}=1.800 ; \mathrm{Mn}=505 ; \mathrm{Cd}=25$; $\mathrm{Cr}=645 ; \mathrm{Ni}=445 \mathrm{e} \mathrm{Pb}=265$. A condutividade elétrica medida na pasta do LE foi igual a $5,52 \mathrm{mS} \mathrm{cm}^{-1}$, e o $\mathrm{pH}$

Tabela 1. Análise química do solo Argissolo, no local do experimento.

\begin{tabular}{|c|c|c|c|c|c|c|c|c|c|c|}
\hline Profundidade & $\mathrm{P}-$ resina & MO & $\begin{array}{c}\mathrm{pH} \\
\left(\mathrm{CaCl}_{2}\right)\end{array}$ & $\mathrm{Ca}$ & $\mathrm{Mg}$ & K & $\mathrm{H}+\mathrm{Al}$ & SB & $\mathrm{T}$ & V \\
\hline$(\mathrm{cm})$ & $\left(\mathrm{mg} \mathrm{dm}^{-3}\right)$ & $\left(\mathrm{g} \mathrm{kg}^{-1}\right)$ & & - --- & ---- & 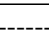 & $\mathrm{mol}_{\mathrm{c}} \mathrm{dr}$ & - & ---- & $(\%)$ \\
\hline $0-20$ & 5 & 13,5 & 4,3 & 5,2 & 1,8 & 1,6 & 24,5 & 8,6 & 33,1 & 26 \\
\hline $20-50$ & 3 & 7,0 & 4,4 & 6,8 & 2,0 & 0,8 & 20,0 & 9,6 & 29,6 & 32 \\
\hline
\end{tabular}

Tabela 2. Teores de óxidos de ferro e alumínio, de nitrogênio, de metais pesados, de boro e de enxofre do Argissolo utilizado para o experimento(1)

\begin{tabular}{|c|c|c|c|c|c|c|c|c|c|c|c|c|c|}
\hline \multirow[t]{2}{*}{ Profundidade } & \multirow[t]{2}{*}{$\mathrm{Fe}_{2} \mathrm{O}_{3}$} & \multirow[t]{2}{*}{$\mathrm{Al}_{2} \mathrm{O}_{3}$} & \multirow[t]{2}{*}{$\mathrm{N}$ total } & \multicolumn{8}{|c|}{ Metais pesados } & \multirow[t]{2}{*}{ B } & \multirow[t]{2}{*}{$\mathrm{S}-\mathrm{SO}_{4}{ }^{-2}$} \\
\hline & & & & $\mathrm{Zn}$ & $\mathrm{Fe}$ & $\mathrm{Cu}$ & $\mathrm{Mn}$ & $\mathrm{Cd}$ & $\mathrm{Cr}$ & $\mathrm{Ni}$ & $\mathrm{Pb}$ & & \\
\hline$(\mathrm{cm})$ & \multicolumn{2}{|c|}{-------- $(\%)$------ } & $\left(\mathrm{mg} \mathrm{kg}^{-1}\right)$ & ---- & ---- & - & ב-..- & - & $\mathrm{dm}^{-}$ & & & & ----- \\
\hline $0-20$ & 2,8 & 2,2 & 1,45 & 0,60 & 37,5 & 0,6 & 30 & 0,05 & 0,06 & 0,20 & 0,97 & 0,05 & 9,0 \\
\hline $20-50$ & 2,0 & 1,9 & 1,05 & 0,40 & 45,6 & 0,4 & 32 & 0,02 & 0,01 & 0,08 & 0,75 & 0,03 & 4,8 \\
\hline
\end{tabular}

${ }^{(1)} \mathrm{F}_{2} \mathrm{O}_{3}$ e $\mathrm{Al}_{2} \mathrm{O}_{3}$ na fração argila; $\mathrm{Zn}, \mathrm{Fe}, \mathrm{Cu}, \mathrm{Mn}, \mathrm{Cd}, \mathrm{Cr}, \mathrm{Ni}$ e Pb extraídos com DTPA; $\mathrm{B}$ extraído com água quente e $\mathrm{S}$ extraído com $\mathrm{CaCl}$. 
em água, igual a 10,2. No processo de tratamento das águas servidas utiliza-se $\mathrm{CaO}$, o que justifica o $\mathrm{pH}$ alcalino e alto teor de $\mathrm{Ca}$ presente no LE. A determinação dos elementos químicos foi efetuada por espectrometria de emissão atômica com plasma acoplado (ICP).

O LE foi analisado também pelos mesmos métodos empregados na análise de solo descritos em Raij \& Quaggio (1983), e os metais pesados foram extraídos pela solução de DTPA (Lindsay \& Norvell, 1978). A composição do LE, expressa em $\mathrm{mmol}_{\mathrm{c}} \mathrm{dm}^{-3}$, foi a seguinte: $\mathrm{Ca}=36,5 ; \mathrm{K}=1,5$ e $\mathrm{Mg}<0,3$, e a concentração de $\mathrm{P}$ foi igual a $21 \mathrm{mg} \mathrm{m}^{-3}$. O LE apresentou a seguinte concentração de metais, extraídos pela solução de DTPA, expressa em mg dm${ }^{-3}: \mathrm{Ni}=66,7 ; \mathrm{Cu}=81,5 ; \mathrm{Zn}=216,0$; $\mathrm{Mn}=35,4$ e Fe $=50,0$.

A parcela experimental foi constituída de cinco linhas de $12 \mathrm{~m}$ de comprimento, com espaços de 1,10 m nas entrelinhas. A área útil da parcela foi constituída pelas três linhas centrais, das quais descontaram-se bordaduras de $1 \mathrm{~m}$ em cada extremidade. O delineamento experimental foi em blocos ao acaso, com três repetições. Os tratamentos, em número de 15 , consistiram de três doses de LE $\left(0,20\right.$ e $40 \mathrm{Mg} \mathrm{ha}^{-1}$, base úmida), combinadas com fertilizantes minerais ( $0, \mathrm{NP}, \mathrm{NK}, \mathrm{PK}$ e NPK) As quantidades de $\mathrm{N}, \mathrm{P}_{2} \mathrm{O}_{5}$ e $\mathrm{K}_{2} \mathrm{O}$, respectivamente de 60 ,

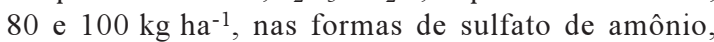
superfosfato triplo e cloreto de potássio, foram calculadas segundo Raij et al. (1985). As doses totais de P e 1/3 das doses de $\mathrm{N}$ e $\mathrm{K}$ foram aplicadas juntamente com o LE no sulco de plantio da cana-de-açúcar, aproximadamente a $30 \mathrm{~cm}$ de profundidade. As mudas, após sua colocação e picação foram cobertas com $5 \mathrm{~cm}$ de terra. As quantidades restantes de $\mathrm{N}$ e $\mathrm{K}$ foram parceladas aos 30 e 45 dias após o plantio, aplicando-se metade delas em cada época, na superfície do solo, ao lado da linha da cana-de-açúcar.

Amostras compostas do solo foram coletadas 146 (30/7/93) dias após o plantio (dap), 272 (2/12/93) dap e $484(5 / 7 / 94)$ dap. Cada amostra composta de solo foi constituída por nove amostras simples retiradas das linhas centrais das parcelas, das camadas de solo de 0 a $20 \mathrm{~cm}$ (nas três épocas), e de 20 a $50 \mathrm{~cm}$ (na última época). As amostras compostas de solo foram secadas ao ar e à sombra, peneiradas em malha de $2 \mathrm{~mm}$, e acondicionadas em caixas de papelão. Nas amostras de solo foram determinados: matéria orgânica, $\mathrm{pH}\left(\mathrm{CaCl}_{2} 0,01 \mathrm{M}\right)$, acidez potencial $(\mathrm{H}+\mathrm{Al}), \mathrm{P}, \mathrm{K}, \mathrm{Ca}$ e $\mathrm{Mg}$; e calcularam-se a soma de bases, a CTC efetiva e a saturação por bases (Raij \& Quaggio, 1983). O S-SO ${ }^{2-}$ foi extraído com solução de $\mathrm{CaCl}_{2}\left(1,5 \mathrm{~g} \mathrm{~L}^{-1}\right)$ e quantificado por turbidimetria (Williams \& Steinberg, 1959). Para a determinação de B do solo seguiu-se o método descrito em Instituto Agronô- mico (1994). Os metais (Cu, Mn, Zn, Cr, Ni, Pb, Fe e Cd) foram extraídos pela solução DTPA (Lindsay \& Norvell, 1978), e quantificados por espectrometria de emissão atômica com plasma acoplado. Os teores totais de óxidos de $\mathrm{Fe}$ e de $\mathrm{Al}$ do solo foram determinados segundo o método escrito em Vettori (1969).

Os resultados foram submetidos à análise de variância, e avaliou-se o efeito da adubação mineral pelo teste de Tukey a $5 \%$ e o efeito das doses de LE, por regressão polinomial. As figuras foram construídas com os teores médios das propriedades do solo, obtidos em razão das doses de LE, incluindo-se os tratamentos sem e com complementação de fertilizante mineral.

\section{Resultados e Discussão}

O LE, que é material alcalino ( $\mathrm{pH}>10)$, aumentou o $\mathrm{pH}$ e reduziu a acidez potencial do solo (Figura 1). A acidez potencial da camada de solo de 0 a $20 \mathrm{~cm}$, nos tratamentos que não receberam LE, variou de 14 a $30 \mathrm{mmol}_{\mathrm{c}} \mathrm{dm}^{-3}$, em razão da época de amostragem. Tal variação é explicada por se tratar de valor médio dos tratamentos que tiveram adição de fertilizantes nitrogenados, fosfatados e potássicos mas não receberam LE. Considerando-se somente a média da testemunha sem LE e sem adição de fertilizantes obtiveram-se $6 \mathrm{mmol}_{\mathrm{c}} \mathrm{dm}^{-3} \mathrm{de} \mathrm{H}+\mathrm{Al}$. Tais resultados de variação da acidez potencial indicam que ocorreu um efeito de acidificação do solo, causada pela adição dos fertilizantes minerais.

A CTC efetiva, a soma de bases e a saturação por bases do solo tiveram seus valores aumentados com o aumento da dose de LE (Figuras 1 e 2). Os efeitos do resíduo no solo foram mais pronunciados aos 272 dap, e já haviam praticamente desaparecido aos 484 dap. O Ca e o $\mathrm{Mg}$, nutrientes contidos no LE, foram adicionados ao solo nas quantidades de 175 e $53 \mathrm{~kg} \mathrm{ha}^{-1}$, respectivamente, na dose do resíduo de $40 \mathrm{Mg} \mathrm{ha}^{-1}$. O teor de Ca extraído do LE pela resina foi de $36,7 \mathrm{mmol}_{\mathrm{c}} \mathrm{dm}^{-3}$, o que corresponde a $6 \%$ do $\mathrm{Ca}$ total contido no LE. O Ca e $\mathrm{Mg}$ contidos no LE, e o Ca contido no superfosfato triplo, motivaram consideráveis aumentos da soma de bases e da saturação de bases do solo, e também do Ca trocável do solo, que em relação à testemunha, aumentou em quatro vezes aos 146 dap, e em oito vezes aos 272 dap, quando foi empregada a maior dose do LE (Figuras 1 e 2). 

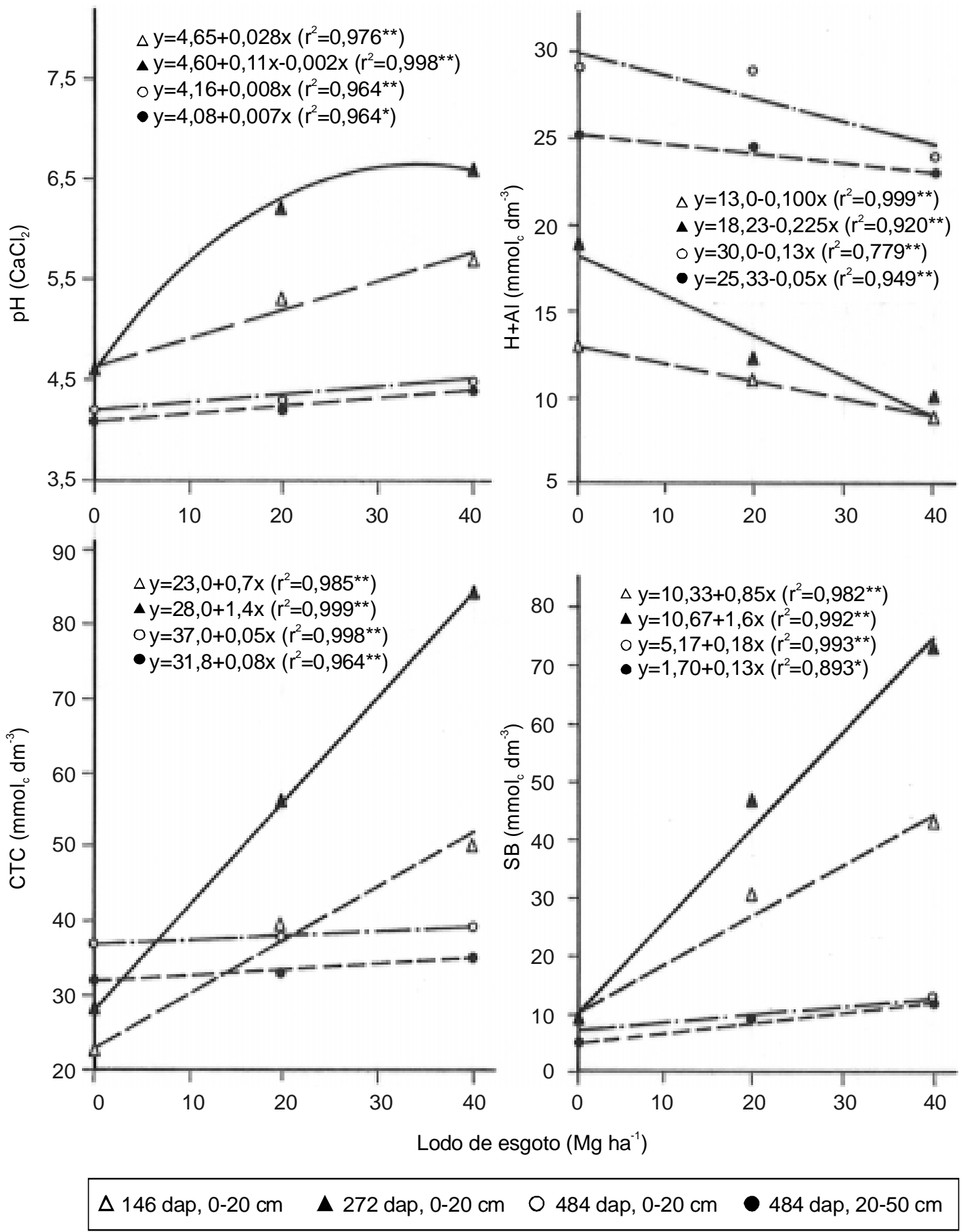

Figura 1. Influência da aplicação de lodo de esgoto na acidez ativa $(\mathrm{pH})$, acidez potencial $(\mathrm{H}+\mathrm{Al})$, capacidade de troca de cátions (CTC) e soma de bases (SB) do solo Argissolo, aos 146, 272 e 484 dias após o plantio (dap). $(*$ e **Significativo a $5 \%$ e a $1 \%$ de probabilidade, respectivamente, pelo teste $\mathrm{F}$ ) 

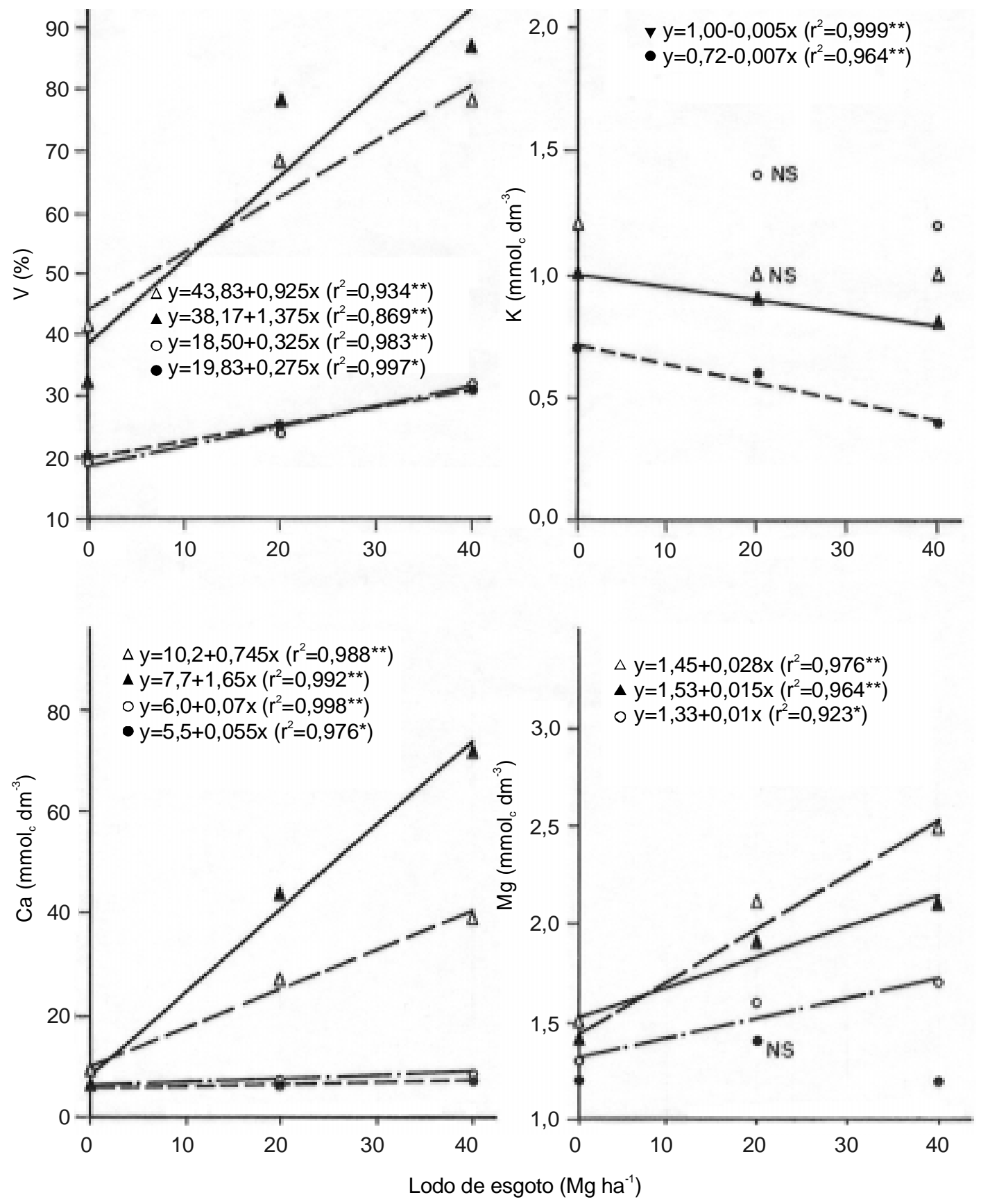

\section{$\Delta 146$ dap, $0-20 \mathrm{~cm} \quad \boldsymbol{\Delta} 272$ dap, 0-20 cm $\quad 484$ dap, 0-20 cm $\quad 484$ dap, 20-50 cm}

Figura 2. Influência da aplicação de lodo de esgoto na saturação por bases (V) e nos teores de potássio (K), cálcio (Ca) e de magnésio (Mg) do solo Argissolo, aos 146, 272 e 484 dias após o plantio (dap). (* e **Significativo a $5 \%$ e a $1 \%$ de probabilidade, respectivamente, pelo teste $\mathrm{F}$ ). 
O LE é um material que tem uma fração orgânica de mais fácil decomposição, pois os seus efeitos no solo, como corretivo da acidez e fornecedor de $\mathrm{Ca}$ e $\mathrm{Mg}$, já haviam praticamente desaparecido aos 484 dap, o que concorda com a observação de Melo et al. $(1994,1997)$.

O LE contém $1,5 \mathrm{~g} \mathrm{~kg}^{-1}$ de $\mathrm{K}$ total, mas apenas $3 \%$ é K trocável e é extraído pela resina. A aplicação de LE causou pequena redução da disponibilidade de K trocável do solo, que era, inicialmente, alto (Figura 2). Com o passar do tempo, houve redução do teor de $\mathrm{K}$ em todos os tratamentos, provavelmente em decorrência da absorção pela cana-de-açúcar, que é exigente neste nutriente. Esse fato evidencia a necessidade de haver aplicação de fertilizante potássico quando se emprega LE e a cultura é exigente em K, como também comentaram Buting (1963) e Boaretto (1986).

A dose de $36 \mathrm{~kg} \mathrm{ha}^{-1}$ de $\mathrm{P}$, na forma de superfosfato triplo, aplicada no fundo do sulco de plantio da canade-açúcar, provocou um aumento no teor de $\mathrm{P}$ do solo, sem, entretanto, alterar a classe de teor, que era muito baixo, segundo os critérios estabelecidos em Raij et al. (1996), em culturas anuais (Tabela 3). Com a aplicação de $66 \mathrm{~kg} \mathrm{ha}^{-1} \mathrm{de} \mathrm{P}$, contido em
$20 \mathrm{Mg} \mathrm{ha}^{-1}$ de LE, o nível de suficiência de P no solo subiu de muito baixo para médio. Com a aplicação de $40 \mathrm{Mg} \mathrm{ha}^{-1}$ de LE o teor de P do solo aumentou ainda mais, mas sem mudar a classe de teor já atingida com a dose de $20 \mathrm{Mg} \mathrm{ha}^{-1}$.

A adição de $40 \mathrm{Mg} \mathrm{ha}^{-1}$ de LE, que contém $132 \mathrm{~kg} \mathrm{ha}^{-1}$ de $\mathrm{S}$, motivou aumento do teor de $\mathrm{S}_{-} \mathrm{SO}_{4}$ do solo amostrado, aos 146 e 272 dap, o que comprova que o resíduo é fonte deste nutriente vegetal (Figura 3). O teor de $\mathrm{S}_{-} \mathrm{SO}_{4}$ do solo, inicialmente baixo na testemunha - segundo critério citado em Kliemann (1987) -, voltou a ser baixo aos 484 dap na profundidade $0-20 \mathrm{~cm}$, independentemente da aplicação do resíduo.

Os teores de $\mathrm{Zn}$ e $\mathrm{Cu}$, que eram inicialmente médios, conforme critério de Raij et al. (1996), passaram a valores considerados altos, quando houve aplicação de LE (Figura 3). Nikitin (1960) e Silva et al. (1998) também verificaram que o LE é fonte de $\mathrm{Zn} \mathrm{e}$ $\mathrm{Cu}$. A aplicação, no solo, de $40 \mathrm{Mg} \mathrm{ha}^{-1}$ de LE, resultou nas adições de $13,8 \mathrm{~kg} \mathrm{ha}^{-1}$ de $\mathrm{Cu}$, e de $27,8 \mathrm{~kg} \mathrm{ha}^{-1} \mathrm{de} \mathrm{Zn}$; entretanto, deste total, apenas 9\% de $\mathrm{Cu}$ e $12 \%$ de $\mathrm{Zn}$ foram extraídos do LE pelo DTPA (Tabela 4).

Tabela 3. Teores de P-resina da camada de 0 a $20 \mathrm{~cm}$ do Argissolo coletada aos 146, 272 e 484 dias após a aplicação do lodo de esgoto $(\mathrm{LE})^{(1)}$.

\begin{tabular}{|c|c|c|c|}
\hline \multirow[t]{2}{*}{ Tratamento } & \multicolumn{3}{|c|}{ Dias após a aplicação do LE } \\
\hline & 146 & 272 & 484 \\
\hline & -------------------- & $\left.\mathrm{g} \mathrm{dm}^{-3}\right)-$ & ----- \\
\hline Testemunha & $2,2 d$ & $4,6 f$ & 5,0 \\
\hline NP & $4,5 \mathrm{~d}$ & $11,6 \mathrm{e}$ & 7,0 \\
\hline NK & $2,0 \mathrm{~d}$ & $5,4 \mathrm{f}$ & 5,0 \\
\hline PK & $4,6 \mathrm{~d}$ & $11,9 \mathrm{e}$ & 7,0 \\
\hline NPK & $5,0 \mathrm{~d}$ & $12,4 \mathrm{e}$ & 7,5 \\
\hline $\mathrm{LE}\left(20 \mathrm{Mg} \mathrm{ha}^{-1}\right)$ & $15,0 \mathrm{bc}$ & $22,5 \mathrm{~d}$ & 7,0 \\
\hline $\mathrm{LE}\left(20 \mathrm{Mg} \mathrm{ha}^{-1}\right)+\mathrm{NP}$ & $18,8 \mathrm{bc}$ & $27,0 \mathrm{~cd}$ & 8,2 \\
\hline $\mathrm{LE}\left(20 \mathrm{Mg} \mathrm{ha}^{-1}\right)+\mathrm{NK}$ & $21,4 b$ & $24,2 \mathrm{~cd}$ & 6,5 \\
\hline $\mathrm{LE}\left(20 \mathrm{Mg} \mathrm{ha}^{-1}\right)+\mathrm{PK}$ & $20,2 b c$ & $26,4 \mathrm{~cd}$ & 8,0 \\
\hline $\mathrm{LE}\left(20 \mathrm{Mg} \mathrm{ha}^{-1}\right)+\mathrm{NPK}$ & $19,2 \mathrm{bc}$ & $27,5 \mathrm{~cd}$ & 8,5 \\
\hline $\mathrm{LE}\left(40 \mathrm{Mg} \mathrm{ha}^{-1}\right)$ & $32,5 \mathrm{a}$ & $38,4 \mathrm{abc}$ & 12,5 \\
\hline $\mathrm{LE}\left(40 \mathrm{Mg} \mathrm{ha}^{-1}\right)+\mathrm{NP}$ & $30,0 \mathrm{ab}$ & $46,0 \mathrm{ab}$ & 9,0 \\
\hline $\mathrm{LE}\left(40 \mathrm{Mg} \mathrm{ha}^{-1}\right)+\mathrm{NK}$ & $35,0 \mathrm{a}$ & $55,4 \mathrm{a}$ & 8,0 \\
\hline $\mathrm{LE}\left(40 \mathrm{Mg} \mathrm{ha}^{-1}\right)+\mathrm{PK}$ & $29,5 \mathrm{ab}$ & $50,4 \mathrm{a}$ & 7,8 \\
\hline $\mathrm{LE}\left(40 \mathrm{Mg} \mathrm{ha}^{-1}\right)+\mathrm{NPK}$ & $28,0 \mathrm{ab}$ & $48,6 \mathrm{ab}$ & 8,5 \\
\hline
\end{tabular}

${ }^{(1)}$ Médias seguidas de mesma letra, na coluna, não diferem estatisticamente entre si pelo teste de Tukey a 5\% de probabilidade. 

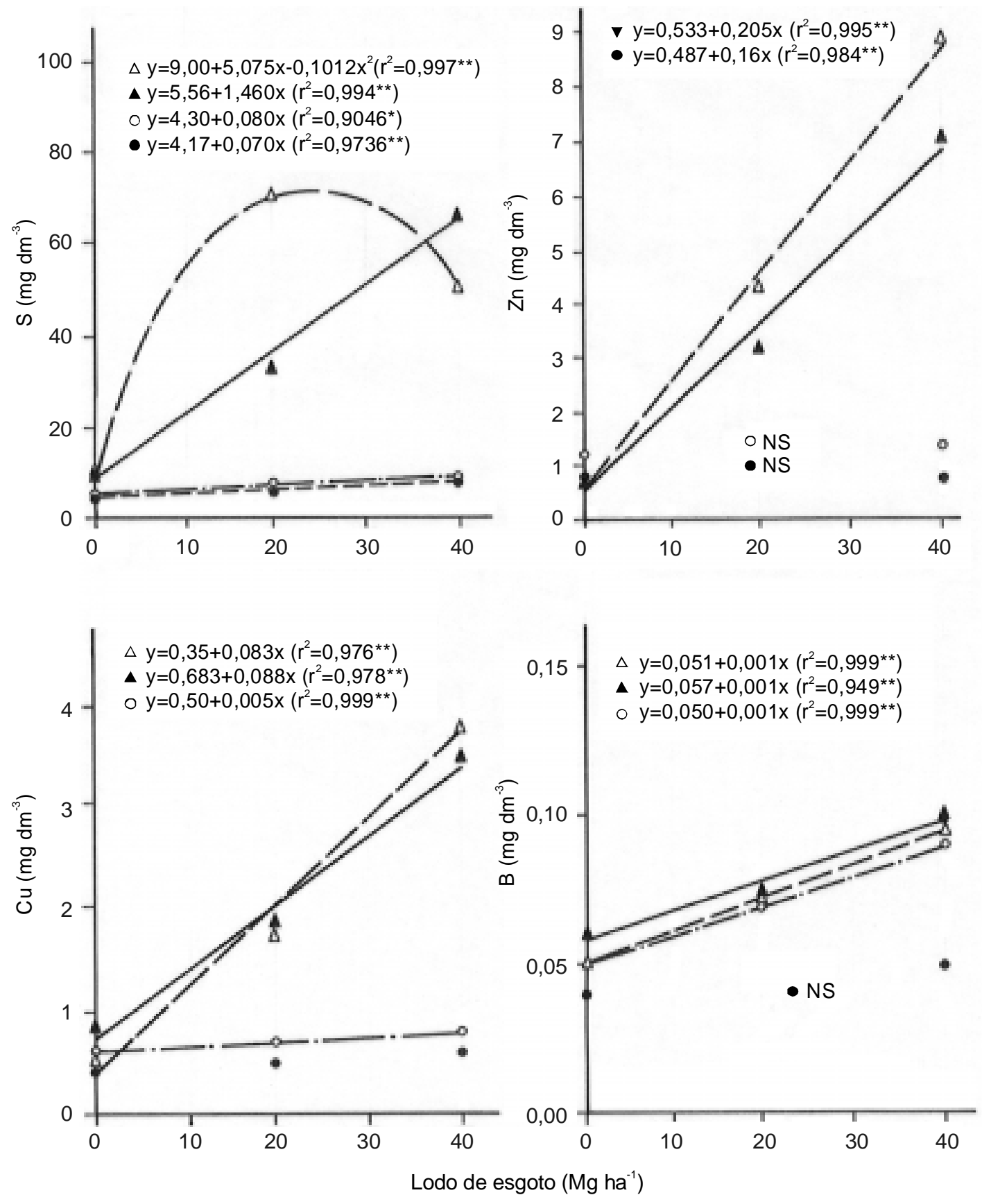

$\Delta 146$ dap, $0-20 \mathrm{~cm} \quad \boldsymbol{\Delta} 272$ dap, $0-20 \mathrm{~cm} \quad$ O 484 dap, $0-20 \mathrm{~cm}$

484 dap, $20-50 \mathrm{~cm}$

Figura 3. Influência da aplicação de lodo de esgoto nos teores de enxofre (S), de zinco $(\mathrm{Zn})$, de cobre $(\mathrm{Cu})$ e de boro (B) do solo Argissolo, aos 146, 272 e 484 dias após o plantio (dap). (* e **Significativo a 5\% e a 1\% de probabilidade, respectivamente, pelo teste $\mathrm{F}$ ). 
Apesar de haver aumento no teor de B do solo, motivado pela aplicação de LE (Figura 3), este permaneceu baixo, segundo Raij et al. (1996), o que indica que o LE é fonte pobre deste micronutriente.

O teor de Fe do solo extraído pelo DTPA, que já era alto no tratamento-testemunha, segundo Raij et al. (1996), não apresentou alteração significativa, apesar de o solo receber $760 \mathrm{~kg} \mathrm{ha}^{-1}$ de Fe quando $40 \mathrm{Mg} \mathrm{ha}^{-1}$ de LE foram aplicadas. O Fe contido no LE é pouco solúvel, pois apenas $0,1 \%$ do teor total foi extraído pelo DTPA.

A aplicação de $40 \mathrm{Mg} \mathrm{ha}^{-1}$ de LE resultou em diminuição média de $5 \mathrm{mg} \mathrm{dm}^{-3}$ no teor de Mn do solo extraído pelo DTPA, efeito que não alterou a classe de teor, que era alto, segundo Raij et al. (1996). O resíduo contém $505 \mathrm{mg} \mathrm{kg}^{-1}$ de Mn, do qual apenas 7\% foi extraído por DTPA. A diminuição do teor de Mn talvez possa ser explicada pelo aumento do $\mathrm{pH}$ do solo, provocado pela adição de LE. Na Tabela 4, constata-se que a concentração de Mn no solo que recebeu $40 \mathrm{Mg} \mathrm{ha}^{-1} \mathrm{de}$ LE estava muito abaixo do teor máximo aceitável.

Se, por um lado, o LE tem efeitos positivos sobre a fertilidade do solo, a aplicação do resíduo pode ser preocupante, por enriquecer o solo em metais pesados $(\mathrm{Ni}, \mathrm{Cd}, \mathrm{Pb}$ e $\mathrm{Cr}$ ) considerados perigosos do ponto de vista ambiental, como relatam Bell et al. (1991).

$\mathrm{O}$ teor de Ni do LE empregado no experimento, igual a $445 \mathrm{mg} \mathrm{kg}^{-1}$, é pouco superior ao limite de $420 \mathrm{mg} \mathrm{kg}^{-1}$, permitido pela legislação americana, que regulamenta os resíduos que podem ter uso agrí- cola (Estados Unidos, 1993). Apesar disso, o teor de Ni extraído do solo pelo DTPA passou de teor inicial de $0,20 \mathrm{mg} \mathrm{dm}^{-3}$ na profundidade de $0-20 \mathrm{~cm}$ (Tabela 2) para $2,5 \mathrm{mg} \mathrm{dm}^{-3}$, em média, quando a dose de $40 \mathrm{Mg} \mathrm{ha}^{-1}$ de LE foi empregada. Este teor está dentro da faixa de variação dos teores médios encontrados nos solos (Rovers et al., 1983). A baixa contaminação do solo com Ni pode ser explicada pelo fato de que apenas $15 \%$ do Ni total do LE é extraído pelo DTPA.

A aplicação, no solo, de $40 \mathrm{Mg}_{\text {ha-1 }}{ }^{-1}$ de LE, provocou aumentos nos teores de $\mathrm{Cd}, \mathrm{Cr}$ e $\mathrm{Pb}$ extraídos do solo pelo DTPA. Os teores iniciais mostrados na Tabela 2 aumentaram para $0,2 \mathrm{mg} \mathrm{dm}^{-3}$ para $\mathrm{Cd} \mathrm{e} \mathrm{Cr}$ e $2,5 \mathrm{mg} \mathrm{dm}^{-3}$ para $\mathrm{Pb}$, pois adicionaram-se ao solo $0,4 \mathrm{~kg} \mathrm{ha}^{-1}$ de Cd, 9,8 $\mathrm{kg} \mathrm{ha}^{-1}$ de Cr e 4,0 $\mathrm{kg} \mathrm{ha}^{-1}$ de $\mathrm{Pb}$. O Cd e o Pb contidos no LE têm baixa solubilidade, ou seja, apenas $20 \%$ destes metais foram extraídos pelo DTPA, o que foi similar aos resultados de Silva et al. (1998).

Com base no que foi acima relatado, pode-se afirmar com segurança que a aplicação de até $40 \mathrm{Mg} \mathrm{ha}^{-1}$ de LE, com concentrações de metais pesados iguais às do utilizado no presente experimento, teve efeito insignificante para o ambiente, pois os teores de $\mathrm{Cd}, \mathrm{Cr}, \mathrm{Ni} \mathrm{e} \mathrm{Pb}$, apesar de sofrerem aumentos, permaneceram dentro da faixa de teores encontrados nos solos do Estado de São Paulo (MattiazzoPrezotto, 1994). Esta afirmação é também amparada pela comparação entre os teores totais determinados no solo que recebeu $40 \mathrm{Mg} \mathrm{ha}^{-1}$ de LE e os teores

Tabela 4. Teores de metais pesados no solo da camada de 0 a $20 \mathrm{~cm}$ que recebeu $40 \mathrm{Mg}^{a^{-1}}$ de lodo de esgoto (LE) aos 272 dias após o plantio (dap) ${ }^{(1)}$.

\begin{tabular}{|c|c|c|c|c|c|c|}
\hline \multirow[t]{2}{*}{ Elemento } & \multirow[t]{2}{*}{ LE } & \multirow[t]{2}{*}{ A } & \multirow{2}{*}{$\begin{array}{l}\mathrm{B} \\
\text { total }\end{array}$} & \multirow{2}{*}{$\begin{array}{c}\mathrm{C} \\
\text { total }\end{array}$} & \multicolumn{2}{|c|}{ Teor total máximo no solo } \\
\hline & & & & & Cottenie (1981) & Pepin \& Coleman (1984) \\
\hline & $\left(\mathrm{mg} \mathrm{kg}^{-1}\right)$ & $\left(\mathrm{kg} \mathrm{ha}^{-1}\right)$ & \multicolumn{2}{|c|}{----- $\left(\mathrm{mg} \mathrm{dm}^{-3}\right)$----- } & \multicolumn{2}{|c|}{ - } \\
\hline $\mathrm{Cd}$ & 25 & 0,4 & 0,5 & 0,15 & 3 & $3-8$ \\
\hline $\mathrm{Ni}$ & 445 & 6,8 & 19,0 & 3,8 & 50 & 100 \\
\hline $\mathrm{Cr}$ & 645 & 9,8 & 40,5 & 10,2 & 100 & $75-100$ \\
\hline $\mathrm{Pb}$ & 265 & 4,0 & 9,8 & 5,0 & 200 & $100-400$ \\
\hline $\mathrm{Cu}$ & 905 & 13,8 & 20,0 & 6,0 & 50 & $60-125$ \\
\hline $\mathrm{Zn}$ & 1.800 & 27,8 & 60,9 & 7,8 & 300 & $70-400$ \\
\hline $\mathrm{Mn}$ & 505 & 7,7 & 80,0 & 60,0 & 400 & $1.500-3.000$ \\
\hline $\mathrm{Fe}$ & 50.000 & 760,0 & - & - & - & \\
\hline
\end{tabular}

${ }^{(1)} \mathrm{LE}$ : teor contido no LE seco; A: teor em $40 \mathrm{Mg} \mathrm{ha}^{-1}$ de LE; B: teor no solo, aos 272 dap; C: teor inicial no solo. 
máximos aceitáveis no solo, segundo Cottenie (1981) e Pepin \& Coleman (1984), mostrados na Tabela 4. Verifica-se que os teores totais de $\mathrm{Cr}, \mathrm{Ni}, \mathrm{Cd}, \mathrm{e} \mathrm{Pb}$, determinados no solo adubado com $40 \mathrm{Mg} \mathrm{ha}^{-1} \mathrm{de}$ LE, são aproximadamente 2, 3, 6 e 10 vezes menores que os níveis máximos admitidos por Cottenie (1981). Entretanto, é necessário ressaltar que a aplicação de LE no mesmo local e por várias vezes pode resultar em aumentos dos teores de $\mathrm{Cr}$, $\mathrm{Ni}$ e $\mathrm{Cd}$ no solo, principalmente, e causar efeitos deletérios nos animais, caso entrem na cadeia trófica. $\mathrm{O} \mathrm{Cd}$ é um dos elementos que devem ser monitorados no solo onde se emprega LE, pois tem grande mobilidade no ambiente, e é o metal mais biodisponível em comparação com os elementos $\mathrm{Cr}, \mathrm{Pb}$ e $\mathrm{Ni}$ (Tyler et al., 1989).

\section{Conclusões}

1. O lodo de esgoto aumenta a fertilidade do solo pela diminuição da acidez, pelo fornecimento de nutrientes, principalmente de $\mathrm{Ca}, \mathrm{P}, \mathrm{S}$ e $\mathrm{Zn}$, e pelo aumento da CTC efetiva.

2. Os efeitos do lodo de esgoto na fertilidade do solo perduram por apenas um ano agrícola.

3. O lodo de esgoto aumenta os teores de metais pesados do solo, mas estes permanecem aquém dos valores considerados perigosos ao ambiente.

\section{Referências}

BELL, P. F.; JAMES, B. R.; CHANEY, R. L. Heavy metal extractability in long-term sewage sludge and metal saltamended soils. Journal of Environmental Quality, Madison, v. 20, p. 481- 486, 1991.

BERTON, R. S.; CAMARGO, O. A.; VALADARES, J. M. A. S. Absorção de nutrientes pelo milho em resposta à adição de lodo de esgoto a cinco solos paulistas. Revista Brasileira de Ciência do Solo, Campinas, v. 13 , p. $187-192,1989$.

BETTIOL, W.; CARVALHO, P. C. T.; FRANCO B. J. D. C. Utilização do lodo de esgoto como fertilizante O Solo, Piracicaba, v. 75, p. 44-54, 1983.

BOARETTO, A. E. Uso de lodo de esgoto como fertilizante. Botucatu : UNESP/FINEP, 1986. 185 p.

BUTING, A. H. Experiments on organic manures. Journal of Agriculture Science, Cambridge, Grã-Bretanha, v. 60, p. $121-140,1963$.
CARVALHO, P. C. T.; BARRAL, M. F. Aplicação de lodo de esgoto como fertilizante. Fertilizantes, Piracicaba, v. 3, p. 1-4, 1981.

COTTENIE, A. Sludge treatment and disposal in relation to heavy metals. In: INTERNATIONAL CONFERENCE ON HEAVY METALS IN THE ENVIRONMENT, Edinburgh, 1981. Proceedings... Edinburgh : CEP Consultants, 1981. p. 167-175.

DIAS, F. L. F. Efeito da aplicação de calcário, lodo de esgoto e vinhaça em solo cultivado com sorgo granífero (Sorghum bicolor L.). Jaboticabal : UNESP, 1994. 74 p. Tese de Doutorado.

ESTADOS UNIDOS. United States Agency Protection Environment. Standard for the use or disposal of sewage sludge. Federal Register, Washington, v. 58, n. 32, p. 9248-9415, Feb. 1993

INSTITUTO AGRONÔMICO (Campinas, SP). Extração de boro pelo cloreto de bário-microondas. In:

Micronutrientes em solos: métodos analíticos adotados pelo IAC. Campinas : IAC/FAPESP, 1994. p. 25-35.

KLIEMANN, H. J. Contribuição ao estudo da disponibilidade de enxofre em solos brasileiros. Piracicaba : ESALQ, 1987. 231 p. Tese de Doutorado.

LINDSAY, W. L.; NORVELL, W. A. Development of a DTPA soil test for zinc, iron, manganese and cooper. Soil Science Society of America Journal, Madison, v. 42, p. 421-428, 1978 .

LOGAN, T. J.; CHANEY, R. Metals. In: PAGE, A. L.; GLEASON, T. L.; SMITH, J. E.; ISKANDAR, J. K.; SOMMERS, L. E. (Ed.). Utilization of municipal wastewater and sludge on land. Riverside : University of California, 1983. p. 235-326.

MATTIAZZO-PREZOTTO, M. E. Comportamento de cobre, cádmio, cromo, níquel e zinco adicionados a solos de clima tropical em diferentes valores de $\mathbf{p H}$. Piracicaba : ESALQ, 1994. 197 p. Tese de Livre Docência.

MELO, W. J.; MARQUES, M. O.; SANTIAGO, G.; CHELLI, R. A.; LEITE, S. A. S. Efeito de doses crescentes de lodo de esgoto sobre as frações da matéria orgânica e CTC de um solo latossolo cultivado com cana-de-açúcar. Revista Brasileira de Ciência do Solo, Campinas, v. 18 , p. $449-455,1994$.

MELO, W. J.; MARQUES, M. O.; SILVA, F. C.; BOARETTO, A. E. Uso agronômico de resíduos urbanos e impactos ambientais. In: CONGRESSO BRASILEIRO DE CIÊNCIA DO SOLO, 26., 1997, Rio de Janeiro. 
Anais... Rio de Janeiro : Sociedade Brasileira de Ciência do Solo, 1997. CD-ROM. Seção 9, Poluição de Solos.

MULCHI, C. L.; BELL, P. F.; ADAMU, C.; CHANEY, R. L. Long term availability of metals in sludge amended acid soils. Journal of Plant Nutrition, New York, v. 10, p. 1149-1161, 1987

NIEUWENHUIZE, J.; POLEY-VOS, C. H.; AKKER, A.; DELFT, W. Comparison of microwave and convention extraction techniques for the determination of metals in soil, sediment and sludge samples by atomic spectrometry. Analyst, Cambridge, Grã-Bretanha, v. 116, p. 347-351, 1991

NIKITIN, A. A. Production and use of trace salts in fertilizers. In: SAUCHELLI, V. (Ed.). The chemistry and technology of fertilizers. New York : Reinhold, 1960 p. $252-278$.

PEPIN, R. G.; COLEMAN, P. Paper mill sludge and ash as soil conditioner. Biocycle, Emmaus, v. 25, p. 52-55, 1984.

RAIJ, B. van; CANTARELLA, H.; QUAGGIO, J. A.; HIROCE, R.; FURLANI, M. C. (Ed.). Recomendações de adubação e calagem para o Estado de São Paulo. 2. ed. Campinas : Instituto Agronômico, 1996. 285 p. (IAC Boletim Técnico, 100).

RAIJ, B. van; QUAGGIO, J. A. Métodos de análises de solos para fins de fertilidade. Campinas : Instituto Agronômico, 1983. 31 p. (IAC. Boletim Técnico, 81).

RAIJ, B. van; SILVA, N. M. da; BATAGLIA, O. C. QUAGGIO, J. A.; HIROCE, R.; CANTARELLA, H.; BELIINAZZI JU'NIOR, R.; DECHEN,A. R.; TRANI, P. E. (Ed.). Recomendações de adubação e calagem para o Estado de São Paulo. Campinas : Instituto Agronômico, 1985. 107 p. (IAC. Boletim Técnico, 100).
ROS, C. O.;AITA, C.; CERETTAM, C. A.; FRIES, M. R. Lodo de esgoto: efeito imediato no milheto e residual na associação aveia-ervilhaca. Revista Brasileira de Ciência do Solo, Campinas, v. 17, p. 257-261, 1991.

ROVERS, H.; CAMARGO, O. C.; VALADARES, J. M. A. S. Níquel total e solúvel em DTPA em solos no Estado de São Paulo. Revista Brasileira de Ciência do Solo, Campinas, v. 7, p. 212-220, 1983.

SARRUGE, J. R.; HAAG, H. P. Análises químicas em plantas. Piracicaba : ESALQ, 1974. $56 \mathrm{p}$

SILVA, F. C. da; BOARETTO, A. E.; BERTON, R. S.; ZOTELLI, H. B.; PEIXE, C. A.; MENDONÇA, E. Canade-açúcar cultivada em solo adubado com lodo de esgoto: nutrientes, metais pesados e produtividade. Pesquisa Agropecuária Brasileira, v. 33, n. 1, p. 1-8, jan. 1998.

TYLER, G.; PAHLSSON, M. B.; BAATH, B. E.; TRANVIK, L. Heavy-metal ecology of terrestrial plants, microorganisms and invertebrates: a review. Water, Air and Soil Pollution, Dordrecht, v. 47, p. 189-215, 1989.

VALADARES, J. M. A. S.; GAL, M.; MILGELGRIN, V.; PAGE, A. L. Some heavy metals in soils treated with sewage sludge: their effects on yield, and their uptake by plants. Journal of Environmental Quality, Madison, v. 12 , p. $49-57,1983$.

VETTORI, L. Métodos de análise do solo. Rio de Janeiro : Ministério da Agricultura, 1969. 24 p. (Boletim Técnico, 7).

WILLIAMS, C. H.; STEINBERG,A. Soil sulphur fractions as chemical indices of available sulphur in some Australian soils. Australian Journal of Agricultural Research, Collingwood, v. 10, p. 340-352, 1959. 